\title{
The Host Cell Recognition and Penetration Apparatus of Staphylococcal Bacteriophages
}

James Kizziah $^{1}$, Keith Manning ${ }^{1}$, Altaira Dearborn ${ }^{2}$ and Terje Dokland ${ }^{1}$

${ }^{1}$ University of Alabama at Birmingham, Birmingham, Alabama, United States, ${ }^{2}$ National Institute of Allergy and Infectious Diseases, Bethesda, Maryland, United States

Staphylococcus aureus is an opportunistic pathogen responsible for a wide range of infections in humans and livestock. Most resistance and virulence factors in $S$. aureus are encoded on mobile genetic elements (MGEs), including bacteriophages, genomic islands and plasmids. Transduction by bacteriophages provides the main means for horizontal transfer of MGEs in S. aureus, which is not naturally transformable and rarely undergoes conjugation. The emergence of virulent, antibiotic resistant strains of $S$. aureus is a considerable public health concern, and has re-ignited interest in the use of bacteriophages for therapeutic use against these pathogens.

Tailed bacteriophages with double-stranded DNA genomes (order Caudovirales) have icosahedral or prolate heads (capsids) with long or short tails attached at one vertex, capped by a baseplate and frequently decorated with one or more tail fibers. The baseplate provides the main point of contact with the host, and is involved in host recognition and adsorption, cell wall penetration and degradation, and DNA ejection. Most staphylococcal bacteriophages bind to wall teichoic acid (WTA), a variable carbohydrate polymer present on the surface of most Gram-positive cells.

$80 \alpha$ is a typical example of a staphylococcal siphovirus, having a $\mathrm{T}=7$ icosahedral capsid and a long, flexuous tail. We previously determined cryo-EM structures of $80 \alpha$ capsids [1,2] and have now determined the structure of the $80 \alpha$ baseplate at $3.7 \AA$ resolution (Fig. 1A) [3]. The $80 \alpha$ baseplate structure has several unique features, including the presence of three tail fiber/receptor binding proteins (Fig. 1B), but also displays striking similarities to other phage baseplates, indicating a mix-and-match strategy for baseplate evolution across the phages of the Firmicutes.

The main receptor binding protein (RBP) of $80 \alpha$ forms six trimers that each consists of a kinked $\alpha$-helical stem domain, a platform domain and two tower domains (Fig. 1C). The $80 \alpha$ RBP is strikingly similar to the RBP from other phages that infect $S$. aureus, even those belonging to different viral families, such as the podovirus P68 [4]. In contrast, phages of Staphylococcus epidermidis or Listeria encode RBPs of a different structure. This suggests that the RBP structure is correlated with the distinct WTA present in different bacterial species [5]. 

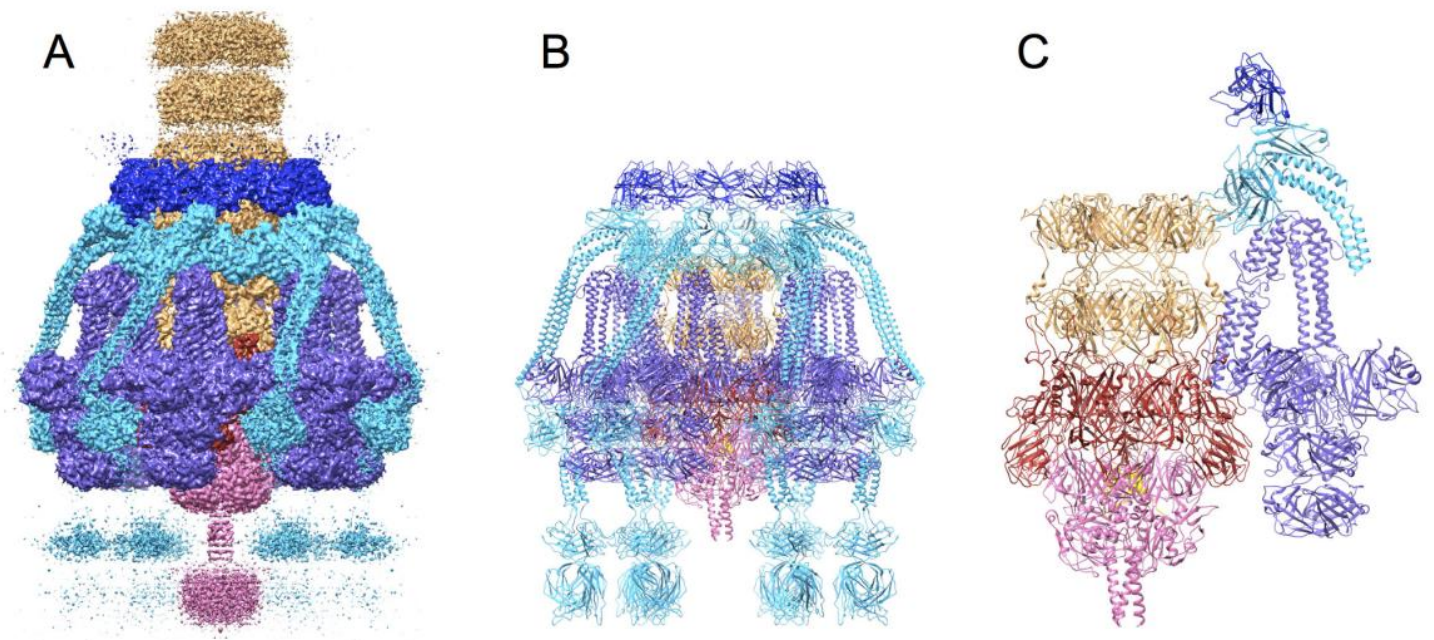

Figure 1. (A) Isosurface representation of cryo-EM reconstruction, and (B) atomic model of the bacteriophage $80 \alpha$ baseplate, colored according to protein: gold, major tail protein (MTP); red, Dit; pink, Tal; purple, RBP; cyan, FibL; blue, FibU. (C) Closeup view of the baseplate core (MTP, Dit, Tal) with all but one of the six peripheral structures (RBP, FibL, FibU) removed [3].

References

[1] Dearborn, A.D., Wall, E.A., Kizziah, J.L., Klenow, L., Parker, L.P., Manning, K.A., Spilman, M.S., Spear, J.M., Christie, G.E., Dokland, T. (2017) eLife 6, e30822

[2] Kizziah, J.L., Manning, K.A., Dearborn, A.D., Wall, E.A., Klenow, L., Hill, R.L., Spilman, M.S., Stagg, S.M., Christie, G.E., Dokland, T. (2017) Viruses 9, E386

[3] Kizziah, J.L., Manning, K.A., Dearborn, A.D., and Dokland, T. (2020) PLoS Pathogens, in press. BioRXiv https://doi.org/10.1101/721746

[4] Hrebik, D., Stverakova, D., Skubnik, K., Füzik, T., Pantucek, R. \& Plevka, P. (2019) Sci. Adv. 5, eaaw7414.

[5] The authors acknowledge John Spear and Nilakshee Bhattacharya at Florida State University (FSU) for cryo-EM data collection support, and Gail E. Christie at Virginia Commonwealth University and José Penadés at Glasgow University for providing strains used in this study. This project was supported by National Institutes of Health grant R01 AI083255 to T.D. The SECM4 resource was supported by NIH grant U24 GM116788 to Kenneth Taylor at FSU. 